\title{
An infrared set-up for continuum thermomechanics
}

\author{
by A. CHRYSOCHOOS (*) and J.C. DUPRE (**)
}

(") Laboratoire de Mécanique et Génie Civil, URA 1214 CNRS, Université Montpellier II, CCo81, pl. E. Bataillon, 34095 Montpellier Cedex, France.

(**) Laboratoire de Mécanique des Solides, Université de Poitiers, 40 av. du Recteur Pineau, 86022 Poitiers, France.

\section{Abstract}

The determination of the constitutive equations of solid materials behaviour is henceforth realized in a thermomechanical framework. Thermodynamics of Irreversible Processes (TIP) allows to introduce internal variables describing the hardening state evolution. This paper presents the performances and the applications of a thermomechanical set-up using infrared thermography elaborated to observe the energetical phenomena accompanying the deformation of the matter. In the case of homogeneous tests, energy balance can be performed to control the validity of behaviour laws.

\section{Nomenclature}

$\dot{\varepsilon} \quad$ strain rate tensor

$\dot{\varepsilon_{\theta}} \quad$ elastic strain rate tensor

$\dot{\varepsilon_{p}} \quad$ plastic strain rate tensor

$w_{\text {oxt }}$ mechanical energy rate

$\dot{w}_{\theta}$ elastic energy rate

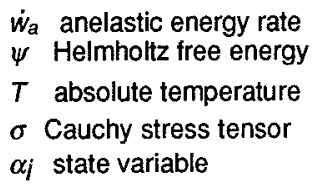

$\dot{w}_{a}$ anelastic energy rate

$T$ absolute temperature

$\alpha_{i}$ state variable

\author{
$\rho$ masse density \\ $\vec{q}$ heat influx vector \\ $D_{1}$ instrinsic dissipation \\ $w_{d}$ dissipated energy \\ $W_{s}$ stored energy of cold work
}

\section{Introduction}

The study of the phenomenological constitutive equations of the solid behaviour is very active in France and particularly in Mecamat (French Group on Mechanics of Materials). The validity of these equations is directly related to the reliabilty of the numerical codes in solid structures. The topic of this paper is a brief description of the theoretical and experimental approach followed to formulate behaviour laws taking into account mechanical, thermal and energetical observations.

After recalling the energy balance form, the definitions of the dissipation, the stored energy rate, the latent heat rate of phase transitions as functions of the state variables are reviewed.

Then a new and improved version of an infrared device associated with a testing machine with numerical commands is presented. This experimental arrangement means that the energy balance in the case of uniaxial and homogeneous solicitations at room temperature can be continuously determined during deformation test. The improvements, in comparison with previous apparatus $[1,2]$, come essentially from technical progress in the storing, processing and visualization of data. This automatic data processing gives the experimentalist both greater accuracy in the determination of the heat sources distribution during deformation processes and a greater flexibility in use.

Then examples of applications are evocated. In the case of homogeneous tests on elastoplastic materials, the evolution of stored energy after deformation during elastic unloading or reversed straining are presented and discussed in comparison with predictions of thermoelastoplastic models developed by [2] within the framework of the Generalized Standard Materials theory (G.S.M. theory [3]). Finally, in the case of heterogeneous experiment, the performances of such an approach are tested to observe birth and evolution of mechanical instabilities: propagation of shear bands, birth of localized necking, cracks growth...

\section{Theoretical background. Energy balance}

\subsection{Mechanical aspects}

In [1] we have shown that in the case of homogeneous and uniaxial solicitations where elastic deformation remains small, most classical kinematic approaches lead to an additive 


\section{http://dx.doi.org/10.21611/qirt.1992.019}

decomposition of strain rate tensor where the elastic and plastic parts ( $\dot{\varepsilon}_{\theta}$ and $\dot{\varepsilon}_{p}$, respectively) can be measured without any doubt. A classical homogeneous test would lead to:

$$
\dot{\varepsilon}=\dot{\varepsilon}_{e}+\dot{\varepsilon}_{p}
$$

Then, the volumic energy rate given by the testing machine for a quasi-static deformation process (ignoring kinetic energy) can be decomposed into:

$$
W_{e x t}=\sigma \dot{\varepsilon}=\sigma \dot{\varepsilon}_{e}+\sigma \dot{\varepsilon}_{p}
$$

We shall call $\dot{w}_{e}$ the rate of volumic energy and $\dot{w}_{a}$ the rate of anelastic energy. The integration of equation (2) leads to:

$$
w_{e x t}=w_{e}+w_{a}
$$

\subsection{Thermodynamical aspects}

Classical results of Thermodynamics of Irreversible Processes are used [4]. An homogeneous volume element of sample is characterized by a set of $n+1$ state variables. Let us take $T=\alpha 0$ as the absolute temperature, $\varepsilon_{e}=\alpha_{1}$ as the elastic strain, and $\left(\alpha_{j}\right)$ as the set of $n-1$ variables describing the hardening state. The specific HELMHOLTZ free energy is classically decomposed in [5]:

$$
\psi\left(T, \varepsilon_{\theta}, \alpha_{j}\right)=\psi_{\theta}\left(T, \varepsilon_{\theta}\right)+\psi_{s}\left(T, \alpha_{j}\right) \quad 2 \leq j \leq n
$$

The 2nd Principle of Thermodynamics leads to the inequality of CLAUSIUS-DUHEM :

$$
\sigma \dot{\varepsilon}-\rho \frac{\partial \psi}{\partial \alpha_{j}} \dot{\alpha}_{j}-\frac{\vec{q}}{T} \overrightarrow{g r a d T} \geq 0 \quad 1 \leq k \leq n
$$

where $\rho$ is the mass density and $\vec{q}$ the heat influx. The intrinsic dissipation can thus be defined:

$$
D_{1}=\sigma \dot{\varepsilon}_{\rho}-\rho \frac{\partial \psi}{\partial \alpha_{j}} \dot{\alpha}_{j} \quad 2 \leq j \leq n
$$

Then, the rate of anelastic energy can be interpreted as the sum of two terms; equations (2) to (6) lead to:

$$
w_{a}=\sigma \dot{\varepsilon}_{p}=D_{1}+\dot{\psi}_{s}
$$

The heat condution equation is written as :

$$
\rho C_{\alpha} \dot{T}-\operatorname{div}[k(g \overrightarrow{r a} d T)]=D_{1}+\rho T \frac{\partial^{2} \psi}{\partial \alpha_{j} \partial T} \dot{\alpha}_{j}+r \quad j=1, \ldots, n
$$

where $r$ symbolizes the external heat supply, $c_{\alpha}=-T \frac{\partial^{2} \psi}{\partial T^{2}}$ is the specific heat when $\alpha_{j}$ remains constant, and $k$ is the conduction tensor. The different heat sources have been gathered in the right hand of the heat conduction equation: one finds respectively the dissipation, the crossing terms providing the thermomechanical couplings (thermoelastic effects, latent heat in case of solid-solid phase transition, etc.), and the external heat supply (radiation, JoulE's effects, etc.).

If we suppose that the external heat supply $r$ is time independent, the conduction tensor $k$ is isotropic and independent of the strain hardening, the specific heat $C_{\alpha}$ is a material constant, and if we note $\theta=T$ - $T_{0}$ then the heat conduction equation can be simplified as:

$$
\rho C_{\alpha} \dot{\theta}-k \Delta \theta=D_{1}+\rho T \frac{\partial^{2} \psi}{\partial \alpha_{j} \partial T} \dot{\alpha}_{j} \quad j=1, . ., n
$$

The variable $T_{0}$ symbolizes the equilibrium temperature field that verifies $-k \Delta T_{0}=r$.

\section{Experimental set-up and calibrations}

\subsection{General description}

The experimental set-up is essentially made of a $100 \mathrm{kN}$ Screw Machine and an infrared camera. The tests and machine are completely controled by micro-computer(figure 1). The infrared camera is built and commercialized by AGEMA Infrared Systems. This device is equipped with the numerization system initially conceived by J.C.CHEZEAUX and B.NAYROLES [6] then developed and improved by the authors. A second micro-computer handles the data 
recording, processing and allows the visualization of the thermal scenes. The camera is placed directly on the crosshead of the testing machine. The thermoregulation is carried out carrefully. The test area must be completely closed. The air is circulated using electric fans and the temperature controled with two heat sources (air conditioner + fan heater ). The video signal quality is improved by anti-radiation screens (polished aluminium) in order to intercept parisitic reflections from the surroundings.

\subsection{Numerized thermal pictures}

They are matrixes of 256 lines per 180 columns numerized on 12 bits. The numerization system allows to record at the beginning of each line, signals like thermal

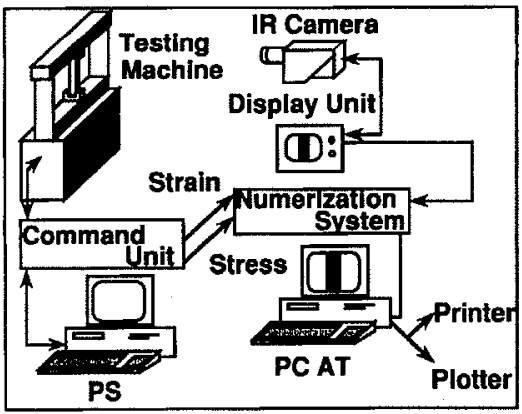

Fig. 1. - Basic sketch of the experimental set-up level and thermal range characterizing the state of the camera, and six electrical signals through two-stage amplifiers, like load and deformation signals, room temperature. In our case (with optional $20^{\circ}$ fixed front lens), the spatial resolution is $d_{x}=(270 \pm 10) 10^{-6} \mathrm{~m}$ and $d_{y}=$

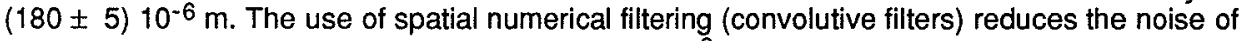
the video signal to temperature variations within $5.10^{-3}{ }^{\circ} \mathrm{C}$ near room temperature.

\subsection{Calibration of the video signal}

A special warming target has been realized to calibrate, near $25^{\circ} \mathrm{C}$, the variations of video signal (Cf. [2] for technical details). The response of the infrared detector (MCT detector, liquid nitrogen cooled) is strongly non linear even in the vicinity of thermal equilibrium. The calibration law has been approximated by a quadratic law. The calibration can be performed in a dynamic way. It does not need to wait for thermal equilibrium and then can be frequently made.

\subsection{Calibration of thermal losses}

In the left hand of the heat conduction equation (9), the laplacian of temperature can be seen. From an experimental point of view, this term is quite difficult to evaluate directly, near thermal equilibrium when thermal gradients are very small, on good thermal conductors like metallic alloys. To determine the volumic losses $-k /\left(\rho C_{\alpha}\right) \Delta \theta$, a numerical estimation of the differential operator is done. This estimation is based on a spatial filtering of data associated with finite differences (see [7] for technical details). A validity control of the method is performed using numerical simulations. In the following example, a heat sources distribution on a parallelepipedic plate and thermal boundary conditions have been chosen. Using analytical or numerical methods (spectral method, finite elements method,etc.), one can determine the evolution of the temperature field on the plate which verifies equation (9). Solutions at instants $t_{k}$ and $t_{k+1}$ are shown on figure 2 and figure 3 . The heat sources distribution at time $t_{k+1 / 2}$ is deduced from both thermal pictures (figure 4) and can be compared with the original distribution On this euristic example, a constant heat supply along each bisecting line has been chosen: $D_{1}(x, y)=s_{0}$ if $0<|x|=|y|<a_{0}$ and $D_{1}(0,0)=2 s_{0}$, where $s_{0}$ et $a_{0}$ are constants and where $(x, y)$ are cartesian coordinates when the origin of the frame is the center of the plate.

\section{Examples of applications}

\subsection{Energetical validity of thermomechanical behaviour laws}

To describe the mechanical behaviour the G.S.M. formalism can be used. In general, models can be developed which give results in good agreement with experiments. Constituted of a superposition of classical models taking into account the isotropic, linear and non linear kinematic hardening rules, these models can predict energy balance evolution which can be compared with the experimental one. On figures 5 and 6 , the theoretical and experimental mechanical responses of a duraluminium are shown. On figures 7 and 8 , the energetical responses can be observed. The experimental data show releases of stored energy during reversed straining or after deformation. That kind of phenomenon cannot be predicted by 
classical theory of elastoplasticity. During elastic unloading or after deformation (time independence), it is classically supposed that the hardening state does not change. No dissipation or release of stored energy should appear .

\subsection{Dissipated phenomena assoclated with mechanical instabllities}

Because of the diffusivity, the thermal response of metallic materials generally gives hazy information on the heat source distribution. The recent progresses made in data storing and processing allows us to associate to thermal scenes, energetical charts on the surface of the sample during its transformation. In the case of heterogeneous phenomena, these techniques have been tested to detect mechanical instabilities. For instance during a compression test, shear bands appear emanating from the hole made in the center of a parallelepipedic and thin plate. On figure 9, the distribution of dissipation (divided by the specific heat ) is shown. On figure 10, the birth of local necking is observed on a law carbon steel during tensile test. It has been observed in [8] that the local necking takes place when the dissipation chart becomes heterogeneous and comparison with predictions of CONSIDERE criterion [9] has been made.

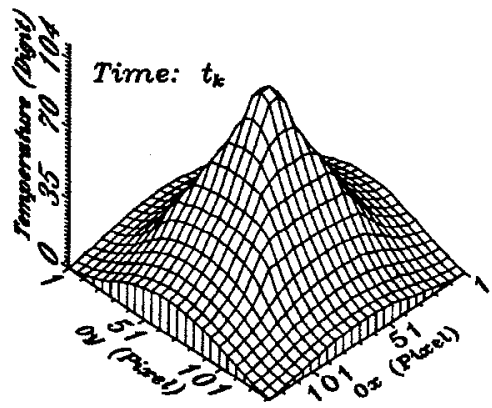

Fig. 2. - Temperature chart, on a parallelepipedic plate at instant $t_{k}$; the thermal boundary conditions are mixted conditions.

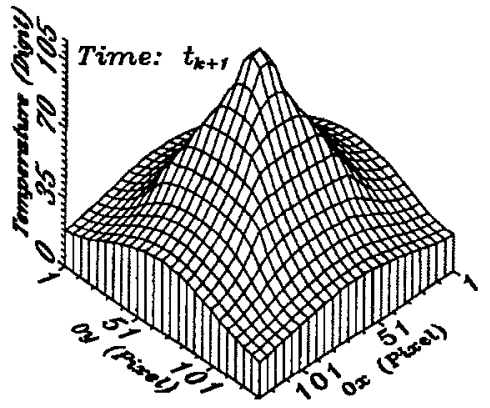

Fig. 3 . - Temperature chart, at instant $t_{k+1}$.

Time: $t_{k+1 / 2}$

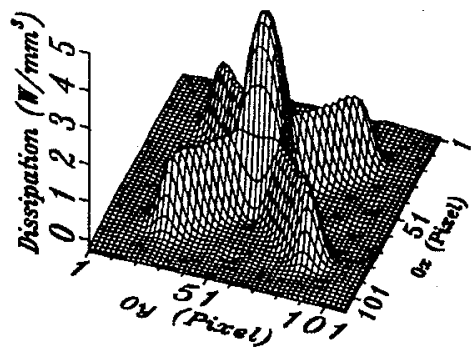

This numerical simulation tries to show that the intuitive passage from temperature to energy is not obvious. The physical good sense can be at fault... The original distribution of dissipation is in this case:

$$
\begin{gathered}
D_{1}(x, y)=s_{0} \text { if } 0<|x|=|y|<a_{0} \\
D_{1}(0,0)=2 s_{0}
\end{gathered}
$$

where $(x=0, y=0)$ localizes the center of the plate.

Fig. 4. - Distribution of heat source deduced from both temperature charts. 


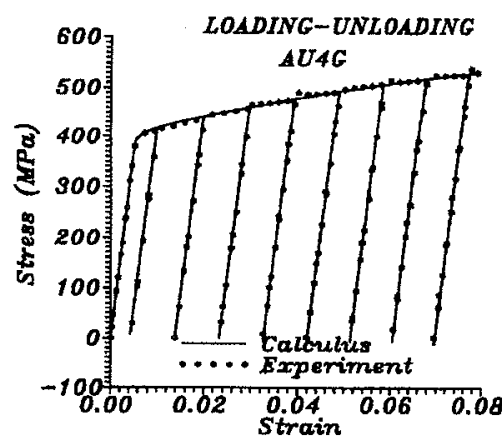

The experimental data are compared to the ones predicted by G.S.M. models within the framework of time rate independent elasto-plasticity. From a mechanical point of view the confrontation between experiment and theory is correct.

Fig. 5:. - Load-unload cycle on a duraluminium sample.

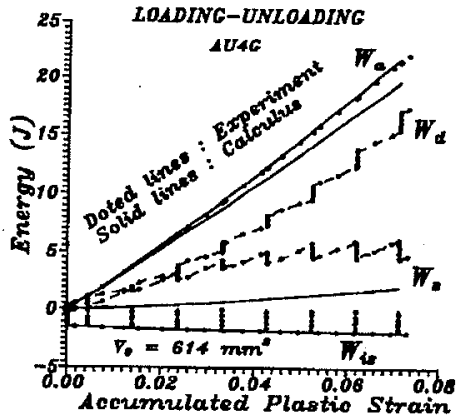

The experimental and predicted anelastic energy curves $\left(W_{\mathrm{a}}\right)$ are superposed. This result comes from the good mechanical validity of the model. Same kind of result is obtained in the case of isentropic energy $\left(W_{\text {is }}\right)$ due to thermoelastic effects. This energy is determined using mechanical data. The dissipated energy $\left(W_{d}\right)$ and the stored energy $\left(W_{S}\right)$ are calculated using the evolution of the thermo. dynamical forces as a function of the state variables. Experimentally, the dissipated energy is deduced from the infrared data. The stored energy is then calculated as the difference between the anelastic energy and the dissipated energy. The order of magnitude of the stored and dissipated energy are quite different. Moreover, release of stored energy can be experimentally observed during elastic unloading. This result cannot be predicted by classical elastoplasticity.

Fig. 7 - Experimental and predicted evolutions of the energy balance during the loadunload cycle defined on figure 5 .

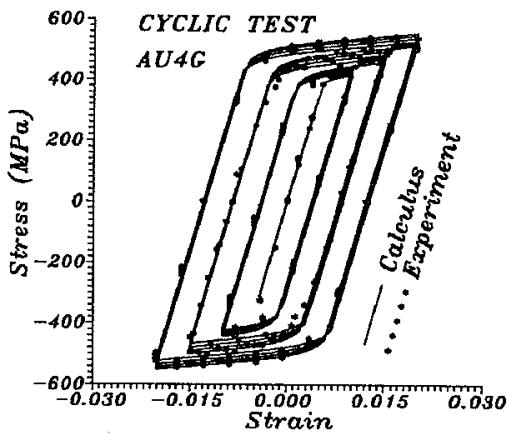

The deformation is controled. Four cycles per each stage have been programmed $( \pm 0,5 \%, \pm 1,0 \%$, $\pm 1,5 \%, \pm 2,0 \%$ ). A weak cyclic hardening can be observed. Once again, the confrontation between experimental and predicted data is correct.

Fig. 6 - Cyclic test on a duratuminium sample.

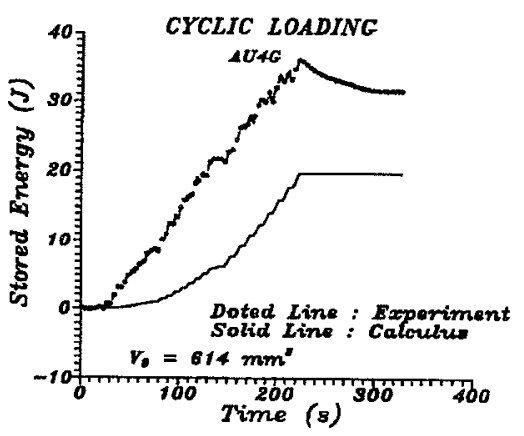

Once again, both evolutions are quite different. A release of stored energy can be experimentally observed after deformation. This result cannot be predicted by a time rate independent formalism. Results presented from fig.5 to fig. 8 show that a model can give correct mechanical simulations without giving for all that good energetical predictions. The mechanical validity of a thermomechanical model does not imply its thermomechanical validity.

Fig. 8. - Evolution of the stored energy during the cyclic test presented on figure 6 . 


\section{http://dx.doi.org/10.21611/qirt.1992.019}

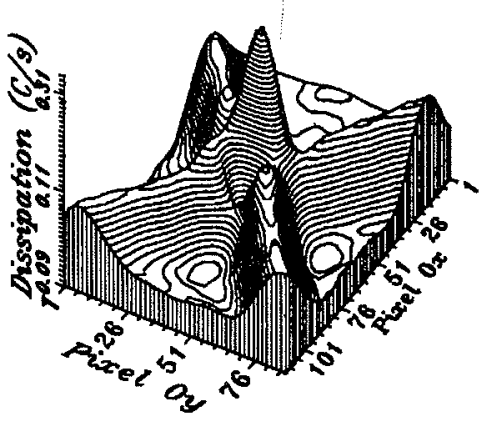

In the center of a parallelepipedic plexiglass plate a hole is realized. A compression test on this plate is perfomed. During the transformation the dissipative effects in the shear bands emanating from the hole can be observed. The stroke velocity of the testing

temperature variations are below $3^{\circ} \mathrm{C}$ during the test.

Fig. 9. - Propagation of shear bands.

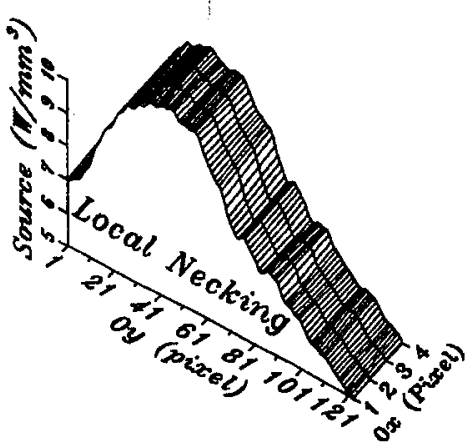

Tensile tests are performed on low carbon steel samples. As long as the mechanical test remains homogeneous, the distribution of mechanical (dissipated) energy given by the testing machine to deform the matter is constant in the gauge length of the sample. The birth of instability (localization of deformation) leads to heterogeneous dissipation charts. Tests have shown that the energetical effects associated to localized necking appear at lower strain than the geometrical (mechanical) ones.

Fig. 10 - Localized necking.

\section{Concluding remarks}

The infrared thermography is a fundamental supplementary asset to study the thermomechanical couplings in solids. This experimental tool can be used with benefit in the case of quasi-static transfomation at room temperature :

- validity controls of classical thermomechanical behaviour laws,

- detection of birth and propagation of mechanical instabilities.

But this domain of applications is in fact a short part of the investigations field that will be approached in the coming years. The behaviour of materials under thermal and mechanical loading paths is always treated by assuming that the sample temperature and the control temperature are identical. This classical approximation masks completely phenomena like phase transitions. Moreover, the constant progresses performed in the field of IR techniques (CCD camera) should allow the observation of thermomechanical couplings during dynamic solicitation. These couplings take a great part in the propagation mode of instabilities.

\section{REFERENCES}

[1] CHRYSOCHOOS (A.). Bilan énergétique en élastoplasticité grandes déformations. J. de Méc. théo. et appl.,Vol.4, n॰5,1985, p.589-614.

[2] BELMAHJOUB (F.). Comportement thermomécanique de materiaux métalliques sous divers trajets de chargement uniaxe. Thèse 1990, 183 pages, Montpellier.

[3] HALPHEN (B.), NGUYEN (Q.S.). Sur les matériaux standards généralisés. J. de Mécanique, Vol.14, 1975 , p. 39-63.

[4] GERMAIN (P.). Cours de mécanique des milieux continus. T1, 1973, 417 pages, Masson et Cie Ed.

[5] LEMAITRE (J.) et CHABOCHE (J.L.).Mécanique des Matériaux solides - 1985, 532 pages, Dunod Ed.

[6] NAYROLES (B.), BOUC (R.), CAUMON (H) et CHEZEAUX (J.C.). Téléthermographie infrarouge et mécanique des structures . Int. J. Engng Sci, 1981, p. 929-947.

[7] DUPRE (J.C.). Traitement et analyse d'images pour la mesure de grandeurs cinématiques, (..), et pour l'étude de couplages thermomécaniques. Thèse, 1992, Poitiers, 254 pages.

[8] CHRYSOCHOOS (A.). Approche énergétique des lois de comportement; relations microstructures propriétés. Rapport de fin de contrat IRSID, 1992.

[9] CONSIDERE (A.). Instabilités plastiques. Ann. des Ponts et Chaussées, Vol. 9, 574, 1885, p.6. 